\title{
Boolean algebras by length recognizability
}

\author{
Didier Caucal and Chloé Rispal ${ }^{1}$ \\ 1 CNRS, LIGM, University Paris-East, France \\ caucal@u-pem.fr and rispal@u-pem.fr
}

\begin{abstract}
We present a simple approach to define Boolean algebras on languages. We proceed by inverse deterministic and length-preserving morphisms on automata whose vertices are words. We give applications for context-free languages and context-sensitive languages.
\end{abstract}

\section{Introduction}

The family of regular languages is closed under many operations. Those closure properties give an easy way to work with this family and specially the closure under Boolean operations. Some of these Boolean closure properties are not satisfied at the next level of the Chomsky hierarchy: the family of context-free languages is not closed under complementation and intersection, and the subfamily of deterministic context-free languages is not closed under union and intersection. A standard way to get Boolean algebras is by recognizability by inverse morphism. This notion has been extended to many finite structures (see [9] among others) and also to infinite automata [4].

An automaton is a set of labeled edges with some initial and final vertices. A morphism $f$ from an automaton $G$ into an automaton $H$ is a mapping from the vertices of $G$ to the vertices of $H$ such that for any edge $s \stackrel{a}{\longrightarrow} t$ of $G, f(s) \stackrel{a}{\longrightarrow} f(t)$ is an edge of $H$ and for $s$ initial/final in $G, f(s)$ is initial/final in $H$. The recognizability by an automaton $H$ according to an automata family $\mathcal{F}$ is defined as the set of languages accepted by the automata of $\mathcal{F}$ that can be mapped by morphism into $H$.

A good way to obtain Boolean algebras of context-free languages is by structural recognizability [4]. Considering a family of automata such that each labeled transition $\stackrel{a}{\longrightarrow}$ is a binary relation on a set $\mathcal{R}$, the morphism has to be a relation of $\mathcal{R}$. This structural notion, together with a natural notion of determinism on morphisms defines Boolean subalgebras of many language families. Nevertheless, those Boolean algebras can be too restrictive. For instance, the set of visibly pushdown languages [1] can not be obtained by structural recognizability.

In this paper, we consider the length recognizability for automata whose vertices are words: the morphisms are still deterministic but we replace the structural condition by the length-preserving property. We define natural conditions on automata families such that this length recognizability defines Boolean subalgebras. The closure under intersection is given by the length synchronization, a natural and usual parallelization operation on word automata. To get the closure under difference, we introduce a new operation: the length superposition. When an automata family is closed under these two operations and under simple conditions, we get a Boolean algebra of languages accepted by automata which are deterministically length recognized by an unambiguous automaton (see Theorems 16 and 17). We give applications for sub-families of context-free languages and of contextsensitive languages. In particular, the family of visibly pushdown languages can be defined by length recognizability. 


\section{Word automata}

We consider finite and infinite automata having words as vertices. In this section, we give basic notations and definitions, and recall the notions of determinism and unambiguity.

Let $N, T$ be countable sets of symbols called respectively non-terminals and terminals. We take a set $C=\{\iota, o\}$ of two colors.

A word automaton $G$ is a subset of $N^{*} \times T \times N^{*} \cup C \times N^{*}$ of vertex set

$$
V_{G}=\{u \mid \exists a, v(u, a, v) \in G \vee(v, a, u) \in G\} \cup\{u \mid \exists c \in C(c, u) \in G\}
$$

such that the following sets are finite:

$$
\begin{aligned}
N_{G}=\left\{x \in N \mid \exists u, v \in N^{*} u x v \in V_{G}\right\} & \text { the set of non-terminals of } G, \\
T_{G}=\{a \in T \mid \exists u, v(u, a, v) \in G\} & \text { the set of terminals or labels of } G .
\end{aligned}
$$

We denote by $I_{G}=\{s \mid(\iota, s) \in G\}$ the set of initial vertices and by $F_{G}=\{s \mid(o, s) \in G\}$ the set of final vertices of $G$. Any triple $(s, a, t) \in G$ is an edge labeled by a from source $s$ to goal $t$; it is also denoted by $s \stackrel{a}{\longrightarrow}_{G} t$ i.e. $\stackrel{a}{\longrightarrow}_{G}=\left\{(s, t) \mid s \stackrel{a}{\longrightarrow}_{G} t\right\}$ is the a-transition of $G$. Any couple $(c, s) \in G$ is a vertex $s$ colored by $c \in C$; it is denoted by $c s \in G$ and $\stackrel{c}{\longrightarrow}{ }_{G}=\{(s, s) \mid c s \in G\}$ is the $c$-transition of $G$.

Taking symbols $\mid, \kappa$, and a triple $\left(T_{-1}, T_{0}, T_{1}\right)$ of disjoint finite subsets of $T$, we define the input-driven automaton:

$$
\begin{aligned}
\operatorname{Inp}\left(T_{-1}, T_{0}, T_{1}\right)= & \left\{\left.\left.\right|^{n} \kappa \stackrel{a}{\longrightarrow}\right|^{n+i} \kappa \mid i \in\{-1,0,1\} \wedge a \in T_{i} \wedge n, n+i \geq 0\right\} \\
\cup & \{\iota \kappa\} \cup\left\{\left.o\right|^{n} \kappa \mid n \geq 0\right\} .
\end{aligned}
$$

The automaton $\operatorname{Inp}(\{b\},\{c\},\{a\})$ is represented below.

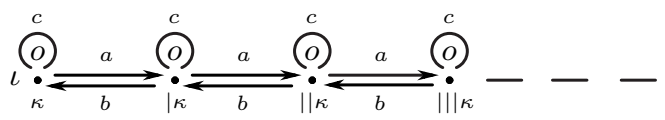

Let $\longrightarrow_{G}$ be the unlabeled edge relation i.e. $s \longrightarrow_{G} t$ if $s \stackrel{a}{\longrightarrow} t$ for some $a \in T$. The accessibility relation $\longrightarrow_{G}^{*}$ is the reflexive and transitive closure under composition of $\longrightarrow_{G}$. A graph $G$ is accessible (resp. co-accessible) from $P \subseteq V_{G}$ if for any $s \in V_{G}$, there is $r \in P$ such that $r \longrightarrow_{G}^{*} s$ (resp. $s \longrightarrow_{G}^{*} r$ ). An automaton $G$ is trimmed if it is accessible from $I_{G}$ and co-accessible from $F_{G}$. The previous automaton is trimmed. The restriction $G_{\mid P}$ of an automaton $G$ to a vertex subset $P$ is the automaton induced by $P$ :

$$
G_{\mid P}=\{(u, a, v) \in G \mid u, v \in P\} \cup\{(c, u) \in G \mid u \in P\} .
$$

The trimmed automaton of $G$ is

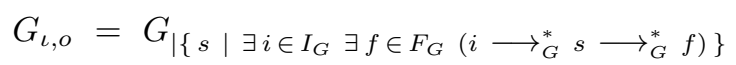

the restriction of $G$ to the vertices accessible from $I_{G}$ and co-accessible from $F_{G}$. Thus $G_{\iota, o}$ is trimmed and $\mathrm{L}\left(G_{\iota, o}\right)=\mathrm{L}(G)$. Similarly, the accessible automaton of $G$ is

$$
G_{\iota}=G_{\mid\left\{s \mid \exists i \in I_{G}\left(i \longrightarrow{ }_{G}^{*} s\right)\right\}} \cdot
$$

Recall that a path is a sequence $s_{0} \stackrel{a_{1}}{\longrightarrow} s_{1} \ldots s_{n-1} \stackrel{a_{n}}{\longrightarrow} s_{n}$ of consecutive transitions; this path leads from the source $s_{0}$ to the goal $s_{n}$ and is labeled by $u=a_{1} \ldots a_{n} \in T^{*}$ and we write $s_{0} \stackrel{u}{\longrightarrow}_{G} s_{n}$. We also write $\iota \stackrel{u}{\longrightarrow} G s, s \stackrel{v}{\longrightarrow} G$ o, $\iota \stackrel{u}{\longrightarrow}_{G} O$ if there exists $i \in I_{G}$ and $f \in F_{G}$ such that we have respectively $i \stackrel{u}{\longrightarrow} G, s \stackrel{v}{\longrightarrow} G f, i \stackrel{u}{\longrightarrow}{ }_{G} f$. A path is accepting if its source is initial and its goal is final. The language accepted by an automaton $G$ is the set $\mathrm{L}(G)=\left\{u \in T^{*} \mid \iota \stackrel{u}{\longrightarrow}_{G} o\right\}$ of labels of its accepting paths. For instance, the previous automaton $\operatorname{Inp}(\{b\},\{c\},\{a\})$ accepts the language

$$
\mathrm{L}(\operatorname{Inp}(\{b\},\{c\},\{a\}))=\left\{\left.u \in\{a, b, c\}^{*}|\forall v \leq u,| v\right|_{a} \geq|v|_{b}\right\}
$$

of prefixes of well-parenthesed words ( $a$ the open parenthesis and $b$ the close one). 
An automaton $G$ is deterministic if it has at most one initial vertex: $\iota s, \iota t \in G \Longrightarrow s=t$, and if for any vertex $r$ and any label $a \in T$, there exists at most one transition starting from $r$ and labeled by $a:\left(r \stackrel{a}{\longrightarrow} G \wedge r \stackrel{a}{\longrightarrow}_{G} t\right) \Longrightarrow s=t$. More generally, an automaton $G$ is unambiguous, if any two accepting paths have distinct labels:

$$
\begin{aligned}
& s_{0} \stackrel{a_{1}}{\longrightarrow} s_{1} \ldots s_{n-1} \stackrel{a_{n}}{\longrightarrow} s_{n} \wedge t_{0} \stackrel{a_{1}}{\longrightarrow} t_{1} \ldots t_{n-1} \stackrel{a_{n}}{\longrightarrow} t_{n} \wedge \iota s_{0}, \iota t_{0}, o s_{n}, o t_{n} \in G \\
& s_{0}=t_{0} \wedge \ldots \wedge s_{n}=t_{n} .
\end{aligned}
$$

The previous automaton $\operatorname{Inp}\left(T_{-1}, T_{0}, T_{1}\right)$ is deterministic. Any deterministic automaton is unambiguous. Here is an unambiguous automaton $U n$ which is not deterministic.

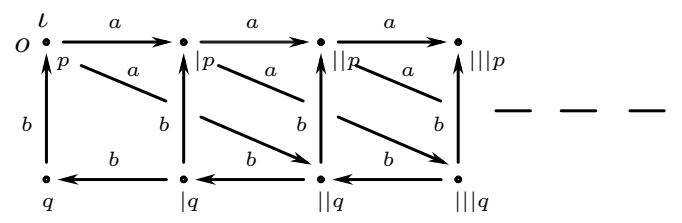

\section{Recognizability}

In order to get Boolean subalgebras of many language families, the recognizability by inverse morphism [5] has been extended to infinite automata [4]. We recall this notion as well as the definition of a deterministic morphism.

A morphism $f$ from an automaton $G$ into an automaton $H$ is a mapping $f: V_{G} \longrightarrow V_{H}$ such that for any $s, t \in V_{G}, a \in T_{G}$ and $c \in C$,

$$
s \stackrel{a}{\longrightarrow} G \Longrightarrow f(s) \stackrel{a}{\longrightarrow}_{H} f(t) \quad \text { and } \quad c s \in G \Longrightarrow c f(s) \in H
$$

we write $G \stackrel{f}{\longrightarrow} H$ or $G \longrightarrow H$ and we say that $G$ is reducible into $H$.

Any word accepted by an automaton is by morphism accepted by the image automaton.

- Lemma 1. Let $G \longrightarrow H$. We have

$$
\mathrm{L}(G) \subseteq \mathrm{L}(H) \quad \text { and } \quad G^{\prime} \longrightarrow H^{\prime} \quad \text { for any } G^{\prime} \subseteq G \text { and } H \subseteq H^{\prime} .
$$

Let us give uniqueness conditions of a morphism between automata.

- Lemma 2. There is at most one morphism from a trimmed automaton into an unambiguous automaton.

\section{Proof.}

Let $G \stackrel{g}{\longrightarrow} H$ and $G \stackrel{h}{\longrightarrow} H$ with $G$ trimmed and $H$ unambiguous.

Let $s$ be any vertex of $G$.

As $G$ is trimmed, there exists $u, v \in T^{*}$ such that $\iota \stackrel{u}{\longrightarrow} G \stackrel{v}{\longrightarrow}$ o. As $g$ and $h$ are morphisms, we have

$$
\iota \stackrel{u}{\longrightarrow} H g(s) \stackrel{v}{\longrightarrow}_{H} O \quad \text { and } \quad \iota \stackrel{u}{\longrightarrow}_{H} h(s) \stackrel{v}{\longrightarrow}_{H} O .
$$

As $H$ is unambiguous, $g(s)=h(s)$.

For families $\mathcal{F}$ of automata, we want to get Boolean subalgebras of

$$
\mathcal{L}(\mathcal{F})=\{\mathrm{L}(G) \mid G \in \mathcal{F}\} .
$$

Recall that a language family $\mathcal{L}$ is a Boolean algebra relative to a language $L \in \mathcal{L}$ if

$$
P \subseteq L \text { and } L-P, P \cap Q \in \mathcal{L} \text { for any } P, Q \in \mathcal{L} .
$$

A first approach is to take an automata family $\mathcal{F}$ and a recognizer $H \in \mathcal{F}$ to define the set of languages accepted by all possible automata of $\mathcal{F}$ which are reducible to $H$ :

$$
\operatorname{Rec}_{\mathcal{F}}(H)=\{\mathrm{L}(G) \mid G \in \mathcal{F} \wedge G \longrightarrow H\} .
$$


For any finite subset $A \subset T$, we define the trimmed and deterministic automaton $\operatorname{Loop}_{A}$ with a unique vertex $\kappa$ and the loops labeled by each letter of $A$ :

$$
\operatorname{Loop}_{A}=\{\kappa \stackrel{a}{\longrightarrow} \kappa \mid a \in A\} \cup\{\iota \kappa, o \kappa\} .
$$

For any family $\mathcal{F}$ of automata labeled in $A$, each automaton is reducible to $\operatorname{Loop}_{A}$ hence

$$
\operatorname{Rec}_{\mathcal{F}}\left(\operatorname{Loop}_{A}\right)=\mathcal{L}(\mathcal{F})
$$

Thus for the family $\mathcal{F}$ in of finite automata, $\operatorname{Rec}_{\mathcal{F} i n}\left(\operatorname{Loop}_{A}\right)$ is the $\operatorname{set} \operatorname{Reg}\left(A^{*}\right)$ of regular languages over $A$ which is a Boolean algebra. This can be extended replacing $\operatorname{Loop}_{A}$ by any finite automaton.

- Proposition 3. For any finite automaton $H, \operatorname{Rec}_{\mathcal{F} i n}(H)=\{L \subseteq \mathrm{L}(H) \mid L$ regular $\}$ is a Boolean algebra relative to $\mathrm{L}(H)$.

However $\mathcal{L}(\mathcal{F})$ is not in general a Boolean algebra. To get Boolean algebras by recognizability, we introduce simple conditions on the morphisms.

In order to preserve by inverse the determinism, we say that a morphism $G \stackrel{f}{\longrightarrow} H$ is a deterministic morphism and we write $G \stackrel{f}{\longrightarrow} \mathrm{d} H$ if

$$
\begin{aligned}
\iota s, \iota t \in G \wedge f(s) & =f(t) \quad \Longrightarrow \quad s=t \\
r \stackrel{a}{\longrightarrow} s \wedge r \stackrel{a}{\longrightarrow} t \wedge f(s)=f(t) & \Longrightarrow
\end{aligned}
$$

Note that any morphism from a deterministic automaton is a deterministic morphism:

$$
G \stackrel{f}{\longrightarrow} H \wedge G \text { deterministic } \Longrightarrow G \stackrel{f}{\longrightarrow}{ }_{\mathrm{d}} H .
$$

Any deterministic morphism preserves by inverse determinism and unambiguity.

- Lemma 4. Let $G \stackrel{f}{\longrightarrow}{ }_{\mathrm{d}} H$ with $H$ unambiguous (resp. deterministic).

Then $G$ is unambiguous (resp. deterministic) and

$$
\left(\iota \stackrel{u}{\longrightarrow}_{G} s \wedge u \in \mathrm{L}(G) \wedge o f(s) \in H\right) \quad \Longrightarrow \quad o s \in G \text {. }
$$

\section{Proof.}

Let $G \stackrel{f}{\longrightarrow}$ d $H$ with $H$ unambiguous.

i) Let us check that $G$ is unambiguous.

Let $s_{0} \stackrel{a_{1}}{\longrightarrow} G s_{1} \ldots s_{n-1} \stackrel{a_{n}}{\longrightarrow} G s_{n}$ and $t_{0}{\stackrel{a_{1}}{\longrightarrow}}_{G} t_{1} \ldots t_{n-1}{\stackrel{a_{n}}{\longrightarrow}}_{G} t_{n}$ with $\iota s_{0}, \iota t_{0}, o s_{n}, o t_{n} \in G$. As $f$ is a morphism, $f\left(s_{0}\right){\stackrel{a_{1}}{\longrightarrow}}_{H} f\left(s_{1}\right) \ldots f\left(s_{n-1}\right){\stackrel{a_{n}}{\longrightarrow}}_{H} f\left(s_{n}\right)$ with $\iota f\left(s_{0}\right)$ of $f\left(s_{n}\right) \in H$. And $f\left(t_{0}\right){\stackrel{a_{1}}{\longrightarrow}}_{H} f\left(t_{1}\right) \ldots f\left(t_{n-1}\right){\stackrel{a_{n}}{\longrightarrow}}_{H} f\left(t_{n}\right)$ with $\iota f\left(t_{0}\right), o f\left(t_{n}\right) \in H$.

As $H$ is unambiguous, we have $f\left(s_{0}\right)=f\left(t_{0}\right), \ldots, f\left(s_{n}\right)=f\left(t_{n}\right)$.

As $f$ is a deterministic morphism, we get $s_{i}=t_{i}$ by induction on $0 \leq i \leq n$.

ii) Assume that $H$ is deterministic. Let us check that $G$ is deterministic.

Case 1: let $\iota s, \iota t \in G$.

As $f$ is a morphism, $\iota f(s), \iota f(t) \in H$. As $H$ is deterministic, $f(s)=f(t)$.

As $f$ is a deterministic morphism, $s=t$.

Case 2: let $r \stackrel{a}{\longrightarrow} G$ and $r \stackrel{a}{\longrightarrow} G$ t.

As $f$ is a morphism, $f(r) \stackrel{a}{\longrightarrow} G f(s)$ and $f(r) \stackrel{a}{\longrightarrow} G f(t)$.

As $H$ is deterministic, $f(s)=f(t)$. As $f$ is a deterministic morphism, $s=t$.

iii) Let $s_{0} \stackrel{a_{1}}{\longrightarrow} G s_{1} \ldots{\stackrel{a_{n}}{\longrightarrow}}_{G} s_{n}$ with $\iota s_{0} \in G$, of $\left(s_{n}\right) \in H$ and $a_{1} \ldots a_{n} \in \mathrm{L}(G)$. Let us check that $o s_{n} \in G$.

As $a_{1} \ldots a_{n} \in \mathrm{L}(G)$, there exists $t_{0} \stackrel{a_{1}}{\longrightarrow} t_{1} \ldots{\stackrel{a_{n}}{\longrightarrow}}_{G} t_{n}$ with $\iota t_{0}, o t_{n} \in G$.

Thus $f\left(s_{0}\right){\stackrel{a_{1}}{\longrightarrow}}_{H} f\left(s_{1}\right) \ldots{\stackrel{a_{n}}{\longrightarrow}}_{H} f\left(s_{n}\right)$ and $f\left(t_{0}\right){\stackrel{a_{1}}{\longrightarrow}}_{H} f\left(t_{1}\right) \ldots{\stackrel{a_{n}}{\longrightarrow}}_{H} f\left(t_{n}\right)$ with $\iota f\left(s_{0}\right), \iota f\left(t_{0}\right), o f\left(s_{n}\right), o f\left(t_{n}\right) \in H$.

As $H$ is unambiguous, we have $f\left(s_{i}\right)=f\left(t_{i}\right)$ for every $0 \leq i \leq n$. 
As $f$ is deterministic, we get $s_{i}=t_{i}$ for every $0 \leq i \leq n$. Thus $o s_{n}=o t_{n} \in G$.

When restricting to deterministic morphisms in $\operatorname{Rec}_{\mathcal{F}}(H)$, we get the subfamily

$$
\operatorname{dRec}_{\mathcal{F}}(H)=\left\{\mathrm{L}(G) \mid G \in \mathcal{F} \wedge G \longrightarrow_{\mathrm{d}} H\right\} .
$$

Let $\mathcal{F}_{\text {det }}=\{G \in \mathcal{F} \mid G$ deterministic $\}$ and $\mathcal{F}_{\text {una }}=\{G \in \mathcal{F} \mid G$ unambiguous $\}$.

By (1) and Lemma 4, we have

$$
\begin{aligned}
& \operatorname{dRec}_{\mathcal{F}}(H)=\operatorname{Rec}_{\mathcal{F}_{\text {det }}}(H) \quad \text { for any } H \in \mathcal{F}_{\text {det }} \\
& \operatorname{dRec}_{\mathcal{F}}(H) \subseteq \operatorname{Rec}_{\mathcal{F}_{\text {una }}}(H) \quad \text { for any } H \in \mathcal{F}_{\text {una }} .
\end{aligned}
$$

Thus $\operatorname{dRec}_{\mathcal{F}}\left(\operatorname{Loop}_{A}\right)=\mathcal{L}\left(\mathcal{F}_{\text {det }}\right)$ is not in general a Boolean algebra. We now specialize the previous notions by vertex length restriction.

\section{$4 \quad$ Recognizability by length}

To get Boolean algebras, the recognizability for infinite automata has been used with a structural condition [4]. In the following, we replace it by a length-preserving condition. When the morphisms are deterministic and under simple conditions on the automata family, this gives less restrictive Boolean subalgebras.

A word automaton $G$ is length-deterministic if it satisfies the following two conditions:

$$
\begin{aligned}
\iota s, \iota t \in G \wedge|s| & =|t| \quad \Longrightarrow \quad s=t \\
r \stackrel{a}{\longrightarrow} s \wedge r \stackrel{a}{\longrightarrow} G \wedge|s|=|t| & \Longrightarrow
\end{aligned}
$$

For instance, the structure $(\mathbb{N}, 0,<)$ is described by the length-deterministic automaton:

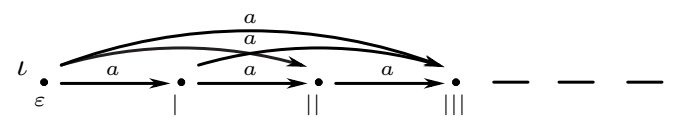

More generally any automaton without two vertices of the same length is length-deterministic. Similarly a word automaton is length-unambiguous if it satisfies the following condition:

$$
\begin{aligned}
& \left(s_{0} \stackrel{a_{1}}{\longrightarrow} s_{1} \ldots s_{n-1} \stackrel{a_{n}}{\longrightarrow} s_{n} \wedge t_{0} \stackrel{a_{1}}{\longrightarrow} t_{1} \ldots t_{n-1} \stackrel{a_{n}}{\longrightarrow} t_{n} \wedge \iota s_{0}, \iota t_{0}, o s_{n}, o t_{n} \in G\right. \\
& \left.\wedge\left|s_{0}\right|=\left|t_{0}\right| \wedge \ldots \wedge\left|s_{n}\right|=\left|t_{n}\right|\right) \Longrightarrow s_{0}=t_{0} \wedge \ldots \wedge s_{n}=t_{n} .
\end{aligned}
$$

We have the following implications:

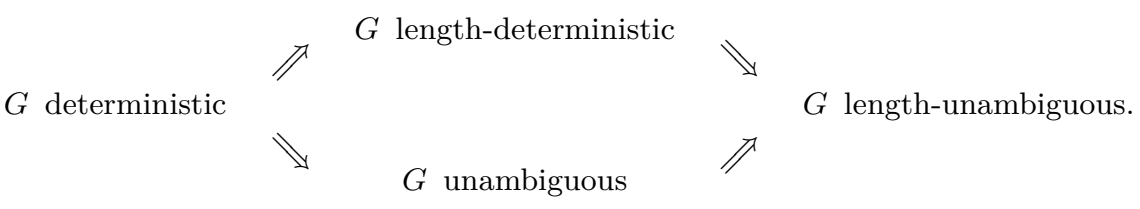

Finally a length-morphism $G \stackrel{f}{\longrightarrow} H$ is a morphism which is length-preserving: $|f(u)|=|u|$ for any $u \in V_{G}$; we write $G \stackrel{f}{\longrightarrow} \ell H$ and we say that $G$ is length-reducible to $H$.

Let us restrict Lemma 2 to length-morphisms.

Lemma 5. There is at most one length-morphism from a trimmed automaton into a length-unambiguous automaton.

Any deterministic length-morphism preserves by inverse the length-determinism and the length-nonambiguity.

- Lemma 6. Let $G \longrightarrow \ell \mathrm{d} H$ with $H$ length-unambiguous (resp. length-deterministic). Then $G$ is length-unambiguous (resp. length-deterministic). 
Let us particularize the subfamilies $\operatorname{Rec}_{\mathcal{F}}(H)$ and $\operatorname{dRec}_{\mathcal{F}}(H)$ by restriction to lengthmorphisms: for any automata family $\mathcal{F}$ and any $H \in \mathcal{F}$, we define

$$
\begin{aligned}
\ell \operatorname{Rec}_{\mathcal{F}}(H) & =\{\mathrm{L}(G) \mid G \in \mathcal{F} \wedge G \longrightarrow \ell H\} \\
\ell \operatorname{Rec}_{\mathcal{F}}(H) & =\{\mathrm{L}(G) \mid G \in \mathcal{F} \wedge G \longrightarrow \ell \mathrm{d} H\} .
\end{aligned}
$$

We have the following inclusions:

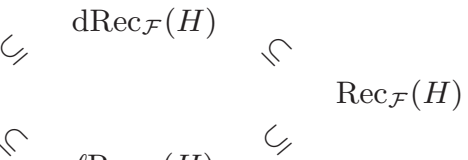

$$
\begin{aligned}
& \ell \operatorname{Rec}_{\mathcal{F}}(H)
\end{aligned}
$$

As $\emptyset \longrightarrow \ell \mathrm{d} H$ and $H \longrightarrow \ell \mathrm{d} H$, we have $\emptyset, \mathrm{L}(H) \in \ell \operatorname{dRec}_{\mathcal{F}}(H)$.

We prove that $\ell \operatorname{dRec}_{\mathcal{F}}(H)$ is a Boolean algebra relative to $\mathrm{L}(H)$ for $H$ unambiguous and $\mathcal{F}$ closed under two simple operations that we introduce now, namely the synchronization by length for the closure under intersection and the superposition by length for the closure under difference.

\section{Synchronization by length}

We define a binary parallelization operation $\|$ on word automata according to the vertex length. We show that $\ell \operatorname{Rec}_{\mathcal{F}}(H)$ is closed under intersection when $H$ is unambiguous and $\mathcal{F}$ is closed under $\|$ (cf. Proposition 9). To get the $\operatorname{closure}$ of $\ell \operatorname{dRec}_{\mathcal{F}}(H)$ under intersection, $\mathcal{F}$ has to be closed under restriction by accessibility from the initial vertices and co-accessibility from the final vertices (cf. Proposition 11).

Let $\Delta_{N}=\left\{(u, v) \in N^{*}|| u|=| v \mid\right\}$ be the set of couples of words over $N$ of same length. The length synchronization is the bijection $\|: \Delta_{N} \longrightarrow(N \times N)^{*}$ defined by

$$
a_{1} \ldots a_{n} \| b_{1} \ldots b_{n}=\left(a_{1}, b_{1}\right) \ldots\left(a_{n}, b_{n}\right) \text { for any } n \geq 0 \text { and } a_{1}, b_{1}, \ldots, a_{n}, b_{n} \in N \text {. }
$$

We also consider the first projection $\pi_{1}$ and the second projection $\pi_{2}$ as the surjective mappings $(N \times N)^{*} \longrightarrow N^{*}$ defined for any $u, v \in N^{*}$ by $\pi_{1}(u, v)=u$ and $\pi_{2}(u, v)=v$. Given word automata $G$ and $G^{\prime}$ with an injection $\phi: N_{G} \times N_{G^{\prime}} \longrightarrow N$, we define their length synchronization $G \|_{\phi} G^{\prime}$ as the following word automaton:

$$
\begin{aligned}
G \|_{\phi} G^{\prime} & =\left\{\phi\left(u \| u^{\prime}\right) \stackrel{a}{\longrightarrow} \phi\left(v \| v^{\prime}\right) \mid u \stackrel{a}{\longrightarrow}{ }_{G} v \wedge u^{\prime} \stackrel{a}{\longrightarrow} G_{G^{\prime}} v^{\prime}\right\} \\
& \cup\left\{\phi\left(u \| u^{\prime}\right) \mid c u \in G \wedge c u^{\prime} \in G^{\prime}\right\} .
\end{aligned}
$$

Since the coding $\phi$ is not essential, it will usually be omitted. Note that

$$
\begin{aligned}
G, G^{\prime} \text { deterministic } & \Longrightarrow G \| G^{\prime} \text { deterministic } \\
V_{G}, V_{G^{\prime}} \text { regular } & \Longrightarrow V_{G} \| V_{G^{\prime}} \text { regular. }
\end{aligned}
$$

As an example, consider the following respective two graphs $G$ and $G^{\prime}$ :
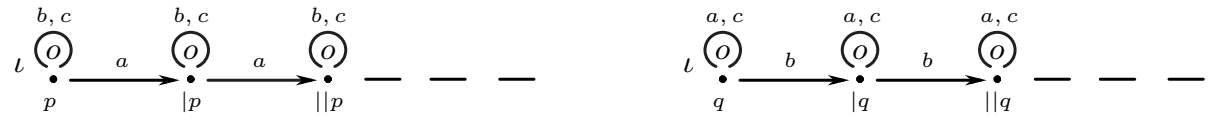

Their length synchronization $G \| G^{\prime}$ is the following graph:

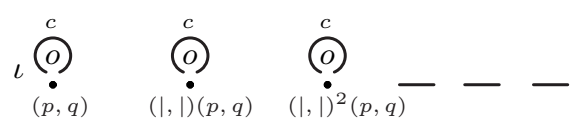

The length synchronization gives the closure under intersection. 
Lemma 7. For any automata $G, G^{\prime}, H$, we have the following properties:

a) $G \| G^{\prime} \stackrel{\pi_{1}}{\longrightarrow} \ell$ and $G \| G^{\prime} \stackrel{\pi_{2}}{\longrightarrow} \ell G^{\prime}$,

b) $\mathrm{L}\left(G \| G^{\prime}\right) \subseteq \mathrm{L}(G) \cap \mathrm{L}\left(G^{\prime}\right)$,

c) if $G \longrightarrow \longrightarrow_{\ell} H$ and $G^{\prime} \longrightarrow_{\ell} H$ and $H$ unambiguous then $\mathrm{L}\left(G \| G^{\prime}\right)=\mathrm{L}(G) \cap \mathrm{L}\left(G^{\prime}\right)$.

Proof.

i) Let us check that $G \| G^{\prime} \stackrel{\pi_{1}}{\longrightarrow} G_{\ell}$.

Let $u\left\|u^{\prime} \stackrel{a}{\longrightarrow} G\right\| G^{\prime} v \| v^{\prime}$. Thus $\pi_{1}\left(u \| u^{\prime}\right)=u \stackrel{a}{\longrightarrow} G=\pi_{1}\left(v \| v^{\prime}\right)$.

Let $c\left(u \| u^{\prime}\right) \in G \| G^{\prime}$. Thus $c \pi_{1}\left(u \| u^{\prime}\right)=c u \in G$.

Finally $\left|u \| u^{\prime}\right|=|u|$. Similarly we check that $G \| G^{\prime} \stackrel{\pi_{2}}{\longrightarrow} G_{\ell}^{\prime}$.

ii) Let us check that $\mathrm{L}\left(G \| G^{\prime}\right) \subseteq \mathrm{L}(G) \cap \mathrm{L}\left(G^{\prime}\right)$.

Let $a_{1} \ldots a_{n} \in \mathrm{L}\left(G \| G^{\prime}\right)$ for some $n \geq 0$ and $a_{1}, \ldots, a_{n} \in T$.

There exists $s_{0}, \ldots, s_{n}$ such that $s_{0}{\stackrel{a_{1}}{\longrightarrow}}_{G \| G^{\prime}} s_{1} \ldots s_{n-1}{\stackrel{a_{n}}{\longrightarrow}}_{G \| G^{\prime}} s_{n}$ with $\iota s_{0}, o s_{n} \in G \| G^{\prime}$. There exists $u_{0}, u_{0}^{\prime}, \ldots, u_{n}, u_{n}^{\prime} \in N^{*}$ such that $s_{i}=u_{i} \| u_{i}^{\prime}$ for every $0 \leq i \leq n$.

Thus $u_{0}{\stackrel{a_{1}}{\longrightarrow}}_{G} u_{1} \ldots u_{n-1}{\stackrel{a_{n}}{\longrightarrow}}_{G} u_{n}$ and $u_{0}^{\prime}{\stackrel{a_{1}}{\longrightarrow} G^{\prime}} u_{1}^{\prime} \ldots u_{n-1}^{\prime}{\stackrel{a_{n}}{\longrightarrow}}_{G^{\prime}} u_{n}^{\prime}$ with $\iota u_{0}, o u_{n} \in G$ and $\iota u_{0}^{\prime}, o u_{n}^{\prime} \in G^{\prime}$. Hence $a_{1} \ldots a_{n} \in \mathrm{L}(G) \cap \mathrm{L}\left(G^{\prime}\right)$.

iii) Let $G \stackrel{f}{\longrightarrow} \ell$ and $G^{\prime} \stackrel{f^{\prime}}{\longrightarrow} \ell H$ with $H$ unambiguous.

Let $a_{1} \ldots a_{n} \in \mathrm{L}(G) \cap \mathrm{L}\left(G^{\prime}\right)$ for some $n \geq 0$ and $a_{1}, \ldots, a_{n} \in T$.

There exists $u_{0} \ldots, u_{n} \in N^{*}$ such that $u_{0}{\stackrel{a_{1}}{\longrightarrow}}_{G} u_{1}, \ldots, u_{n-1}{\stackrel{a_{n}}{\longrightarrow}}_{G} u_{n}$ with $\iota u_{0}, o u_{n} \in G$. There exists $u_{0}^{\prime} \ldots, u_{n}^{\prime} \in N^{*}$ such that $u_{0}^{\prime}{\stackrel{a_{1}}{\longrightarrow}}_{G^{\prime}} u_{1}^{\prime}, \ldots, u_{n-1}^{\prime}{\stackrel{a_{n}}{\longrightarrow} G^{\prime}}_{n}^{\prime}$ with $\iota u_{0}^{\prime}, o u_{n}^{\prime} \in G^{\prime}$. Thus $f\left(u_{0}\right){\stackrel{a_{1}}{\longrightarrow}}_{H} f\left(u_{1}\right), \ldots, f\left(u_{n-1}\right){\stackrel{a_{n}}{\longrightarrow}}_{H} f\left(u_{n}\right)$ with $\iota f\left(u_{0}\right), o f\left(u_{n}\right) \in H$.

Furthermore $f^{\prime}\left(u_{0}^{\prime}\right){\stackrel{a_{1}}{\longrightarrow}}_{H} f^{\prime}\left(u_{1}^{\prime}\right), \ldots, f^{\prime}\left(u_{n-1}^{\prime}\right){\stackrel{a_{n}}{\longrightarrow}}_{H} f^{\prime}\left(u_{n}^{\prime}\right)$ with $\iota f^{\prime}\left(u_{0}^{\prime}\right), o f^{\prime}\left(u_{n}^{\prime}\right) \in H$.

As $H$ is unambiguous, $f\left(u_{0}\right)=f^{\prime}\left(u_{0}^{\prime}\right), \ldots, f\left(u_{n}\right)=f^{\prime}\left(u_{n}^{\prime}\right)$.

As $f, f^{\prime}$ are length-preserving, $\left|u_{0}\right|=\left|u_{0}^{\prime}\right|, \ldots,\left|u_{n}\right|=\left|u_{n}^{\prime}\right|$.

So $u_{0}\left\|u_{0}^{\prime}{\stackrel{a_{1}}{\longrightarrow}}_{G \| G^{\prime}} u_{1}\right\| u_{1}^{\prime} \ldots u_{n-1}\left\|u_{n-1}^{\prime}{\stackrel{a_{n}}{\longrightarrow}}_{G \| G^{\prime}} u_{n}\right\| u_{n}^{\prime}$ with $\iota u_{0}\left\|u_{0}^{\prime}, o u_{n}\right\| u_{n}^{\prime} \in G \| G^{\prime}$. Finally $a_{1} \ldots a_{n} \in \mathrm{L}\left(G \| G^{\prime}\right)$.

Let us give basic properties on the vertices of length synchronized automata.

- Lemma 8. Let $G \stackrel{f}{\longrightarrow} H$ and $G^{\prime} \stackrel{f^{\prime}}{\longrightarrow}{ }_{\ell} H$. We have

a) $\left(u \| u^{\prime}\right.$ vertex of $\left(G \| G^{\prime}\right)_{\iota}$ and $H$ length-deterministic $) \quad \Longrightarrow \quad f(u)=f^{\prime}\left(u^{\prime}\right)$

b) $\left(u \| u^{\prime}\right.$ vertex of $\left(G \| G^{\prime}\right)_{\iota, o}$ and $H$ length-unambiguous $) \quad \Longrightarrow \quad f(u)=f^{\prime}\left(u^{\prime}\right)$.

\section{Proof.}

i) Let $u \| u^{\prime}$ be a vertex of $\left(G \| G^{\prime}\right)_{\iota}$ with $H$ length-deterministic.

Let us show that $f(u)=f^{\prime}\left(u^{\prime}\right)$.

There exists $\left(u_{0} \| u_{0}^{\prime}\right){\stackrel{a_{1}}{\longrightarrow}}_{G \| G^{\prime}}\left(u_{1} \| u_{1}^{\prime}\right) \ldots{\stackrel{a_{n}}{\longrightarrow}}_{G \| G^{\prime}}\left(u_{n} \| u_{n}^{\prime}\right)$ such that $\iota\left(u_{0} \| u_{0}^{\prime}\right) \in G \| G^{\prime}$ and $\left(u, u^{\prime}\right)=\left(u_{n}, u_{n}^{\prime}\right)$.

So $u_{0}{\stackrel{a_{1}}{\longrightarrow}}_{G} u_{1} \ldots{\stackrel{a_{n}}{\longrightarrow}}_{G} u_{n}$ and $u_{0}^{\prime}{\stackrel{a_{1}}{\longrightarrow}}_{G^{\prime}} u_{1}^{\prime} \ldots{\stackrel{a_{n}}{\longrightarrow}}_{G^{\prime}} u_{n}^{\prime}$ such that $\iota u_{0} \in G$ and $\iota u_{0}^{\prime} \in G^{\prime}$ with $\left|u_{0}\right|=\left|u_{0}^{\prime}\right|, \ldots,\left|u_{n}\right|=\left|u_{n}^{\prime}\right|$.

Thus $f\left(u_{0}\right){\stackrel{a_{1}}{\longrightarrow}}_{H} f\left(u_{1}\right) \ldots{\stackrel{a_{n}}{\longrightarrow}}_{H} f\left(u_{n}\right)$ and $\iota f\left(u_{0}\right) \in H$.

Furthermore $f^{\prime}\left(u_{0}^{\prime}\right){\stackrel{a_{1}}{\longrightarrow}}_{H} f^{\prime}\left(u_{1}^{\prime}\right) \ldots{\stackrel{a_{n}}{\longrightarrow}}_{H} f^{\prime}\left(u_{n}^{\prime}\right)$ and $\iota f^{\prime}\left(u_{0}^{\prime}\right) \in H$.

For any $0 \leq i \leq n$, we have $\left|f\left(u_{i}\right)\right|=\left|u_{i}\right|=\left|u_{i}^{\prime}\right|=\left|f^{\prime}\left(u_{i}^{\prime}\right)\right|$.

As $H$ is length-deterministic and by induction on $0 \leq i \leq n$, we get that $f\left(u_{i}\right)=f^{\prime}\left(u_{i}^{\prime}\right)$.

In particular $f(u)=f\left(u_{n}\right)=f^{\prime}\left(u_{n}^{\prime}\right)=f^{\prime}\left(u^{\prime}\right)$.

ii) Let $u \| u^{\prime}$ be a vertex of $\left(G \| G^{\prime}\right)_{\iota, o}$ with $H$ length-unambiguous.

Let us show that $f(u)=f^{\prime}\left(u^{\prime}\right)$.

There exists $\left(u_{0} \| u_{0}^{\prime}\right){\stackrel{a_{1}}{\longrightarrow}}_{G \| G^{\prime}}\left(u_{1} \| u_{1}^{\prime}\right) \ldots{\stackrel{a_{n}}{\longrightarrow}}_{G \| G^{\prime}}\left(u_{n} \| u_{n}^{\prime}\right)$ and $0 \leq p \leq n$ such that 
$\iota\left(u_{0} \| u_{0}^{\prime}\right), o\left(u_{n} \| u_{n}^{\prime}\right) \in G \| G^{\prime}$ and $u\left\|u^{\prime}=u_{p}\right\| u_{p}^{\prime}$.

So $u_{0}{\stackrel{a_{1}}{\longrightarrow}}_{G} u_{1} \ldots{\stackrel{a_{n}}{\longrightarrow}}_{G} u_{n}$ and $u_{0}^{\prime}{\stackrel{a_{1}}{\longrightarrow}}_{G^{\prime}} u_{1}^{\prime} \ldots{\stackrel{a_{n}}{\longrightarrow}}_{G^{\prime}} u_{n}^{\prime}$ such that $\iota u_{0}, o u_{n} \in G$ and $\iota u_{0}^{\prime}, o u_{n}^{\prime} \in G^{\prime}$ with $\left|u_{0}\right|=\left|u_{0}^{\prime}\right|, \ldots,\left|u_{n}\right|=\left|u_{n}^{\prime}\right|$.

Thus $f\left(u_{0}\right){\stackrel{a_{1}}{\longrightarrow}}_{H} f\left(u_{1}\right) \ldots{\stackrel{a_{n}}{\longrightarrow}}_{H} f\left(u_{n}\right)$ and $\iota f\left(u_{0}\right), o f\left(u_{n}\right) \in H$.

Furthermore $f^{\prime}\left(u_{0}^{\prime}\right){\stackrel{a_{1}}{\longrightarrow}}_{H} f^{\prime}\left(u_{1}^{\prime}\right) \ldots{\stackrel{a_{n}}{\longrightarrow}}_{H} f^{\prime}\left(u_{n}^{\prime}\right)$ and $\iota f^{\prime}\left(u_{0}^{\prime}\right), o f^{\prime}\left(u_{n}^{\prime}\right) \in H$.

For any $0 \leq i \leq n$, we have $\left|f\left(u_{i}\right)\right|=\left|u_{i}\right|=\left|u_{i}^{\prime}\right|=\left|f^{\prime}\left(u_{i}^{\prime}\right)\right|$.

As $H$ is length-unambiguous, we get $f\left(u_{0}\right)=f^{\prime}\left(u_{0}^{\prime}\right), \ldots, f\left(u_{n}\right)=f^{\prime}\left(u_{n}^{\prime}\right)$.

In particular $f(u)=f\left(u_{p}\right)=f^{\prime}\left(u_{p}^{\prime}\right)=f\left(u^{\prime}\right)$.

Let us apply Lemma 7 (c) to the intersection closure by length recognizability.

- Proposition 9. The language family $\ell \operatorname{Rec}_{\mathcal{F}}(H)$ is closed under intersection when $H$ is unambiguous and $\mathcal{F}$ is closed under $\|$.

This proposition is not suitable for the family $\ell \operatorname{dRec}_{\mathcal{F}}(H)$ because Lemma 7 (a) cannot be extended to deterministic reductions: $G=\{\varepsilon \stackrel{a}{\longrightarrow} 0, \varepsilon \stackrel{a}{\longrightarrow} 1,1 \stackrel{a}{\longrightarrow} 10, \iota \varepsilon, o 0, o 10\}$ is a trimmed and unambiguous automaton but $G \| G \nrightarrow_{\mathrm{d}} G$ since

$$
\begin{aligned}
G \| G=\{\varepsilon \stackrel{a}{\longrightarrow}(0,0), \varepsilon \stackrel{a}{\longrightarrow}(0,1), \varepsilon \stackrel{a}{\longrightarrow}(1,0), \varepsilon \stackrel{a}{\longrightarrow}(1,1), \\
(1,1) \stackrel{a}{\longrightarrow}(1,1)(0,0), \iota \varepsilon, o(0,0), o(1,1)(0,0)\} .
\end{aligned}
$$

Nevertheless $(G \| G)_{\iota, o} \longrightarrow \ell$ d $G$. This property can be generalized.

- Lemma 10. We have $\quad G \longrightarrow_{\ell} H \vee G^{\prime} \longrightarrow_{\ell} H \Longrightarrow G \| G^{\prime} \longrightarrow_{\ell} H$

$$
G \longrightarrow_{\ell \mathrm{d}} H \wedge G^{\prime} \longrightarrow_{\ell \mathrm{d}} H \wedge H \text { length-unambiguous } \Longrightarrow\left(G \| G^{\prime}\right)_{\iota, o} \longrightarrow_{\ell \mathrm{d}} H .
$$

Proof.

If $G \stackrel{f}{\longrightarrow} \ell H$ then by Lemma 7 (a), $G \| G^{\prime}{\stackrel{\pi_{1}}{\longrightarrow}}_{\ell} G \stackrel{f}{\longrightarrow} \ell H$.

If $G^{\prime} \stackrel{f^{\prime}}{\longrightarrow} \ell H$ then by Lemma 7 (a), $G \| G^{\prime} \stackrel{\pi_{2}}{\longrightarrow} \ell G^{\prime} \stackrel{f}{\longrightarrow} \ell H$.

Suppose that $G \stackrel{f}{\longrightarrow} \ell \mathrm{d} H$ and $G^{\prime} \stackrel{f^{\prime}}{\longrightarrow} \ell \mathrm{d} H$ with $H$ length-unambiguous.

Let $K=\left(G \| G^{\prime}\right)_{\iota, o}$. Let us prove that $K \longrightarrow \ell \mathrm{d} H$. We define

$$
\Delta_{f, f^{\prime}}=\left\{u \| u^{\prime} \mid f(u)=f^{\prime}\left(u^{\prime}\right)\right\}
$$

and the mapping

$$
f \times f^{\prime}: \Delta_{f, f^{\prime}} \longrightarrow N^{*} \quad \text { by }\left(f \times f^{\prime}\right)\left(u \| u^{\prime}\right)=f(u) \text { for any } u \| u^{\prime} \in \Delta_{f, f^{\prime}} .
$$

By Lemma 8 (b), we get $K \stackrel{f \times f^{\prime}}{\longrightarrow} \ell$. Let us check that $K \stackrel{f \times f^{\prime}}{\longrightarrow}$ d $H$.

Case 1: Let $\iota\left(u \| u^{\prime}\right), \iota\left(v \| v^{\prime}\right) \in K$ with $\left(f \times f^{\prime}\right)\left(u \| u^{\prime}\right)=\left(f \times f^{\prime}\right)\left(v \| v^{\prime}\right)$.

Thus $\iota u, \iota v \in G$ and $f(u)=f(v)$. As $G \stackrel{f}{\longrightarrow}{ }_{\mathrm{d}} H$, we get $u=v$.

Furthermore $\iota u^{\prime}, \iota v^{\prime} \in H$ and $f^{\prime}\left(u^{\prime}\right)=f(u)=f(v)=f^{\prime}\left(v^{\prime}\right)$.

As $G^{\prime} \stackrel{f^{\prime}}{\longrightarrow}$ d $H$, we get $u^{\prime}=v^{\prime}$. So $u\left\|u^{\prime}=v\right\| v^{\prime}$.

Case 2: Let $\left(u \| u^{\prime}\right) \stackrel{a}{\longrightarrow}_{K}\left(v \| v^{\prime}\right)$ and $\left(u \| u^{\prime}\right) \stackrel{a}{\longrightarrow}_{K}\left(w \| w^{\prime}\right)$ such that

$$
\left(f \times f^{\prime}\right)\left(v \| v^{\prime}\right)=\left(f \times f^{\prime}\right)\left(w \| w^{\prime}\right) .
$$

So $u \stackrel{a}{\longrightarrow}_{G} v$ and $u \stackrel{a}{\longrightarrow}_{G} w$ with $f(v)=f(w)$. As $G \stackrel{f}{\longrightarrow}_{\mathrm{d}} H$, we get $v=w$.

Furthermore $u^{\prime} \stackrel{a}{\longrightarrow} G_{G^{\prime}} v^{\prime}$ and $u^{\prime} \stackrel{a}{\longrightarrow} G_{G^{\prime}} w^{\prime}$ with $f^{\prime}\left(v^{\prime}\right)=f(v)=f(w)=f^{\prime}\left(w^{\prime}\right)$.

As $G^{\prime} \stackrel{f^{\prime}}{\longrightarrow}$ d $H$, we get $v^{\prime}=w^{\prime}$. So $v\left\|v^{\prime}=w\right\| w^{\prime}$.

We say that an automata family $\mathcal{F}$ is closed under $\iota$-restriction if $G_{\iota, o} \in \mathcal{F}$ for any $G \in \mathcal{F}$. Let us apply Lemmas 7 and 10 .

- Proposition 11. The language family $\ell \operatorname{dRec}_{\mathcal{F}}(H)$ is closed under intersection when $H$ is unambiguous and $\mathcal{F}$ is closed under $\|$ and $\iota 0$-restriction. 
Now we study the closure of $\ell \operatorname{dRec}_{\mathcal{F}}(H)$ under the difference operation.

\section{Superposition by length}

We define a binary superposition operation // on word automata according to vertex lengths. When $\mathcal{F}$ is an automata family closed under //, we obtain simple conditions for $\ell \operatorname{dRec}_{\mathcal{F}}(H)$ to be closed under difference (cf. Proposition 15). Then we obtain two general ways to get $\ell \operatorname{dRec}_{\mathcal{F}}(H)$ as a Boolean algebra relative to $\mathrm{L}(H)$ (Theorems 16 and 17).

We say that a word automaton $G$ is $\varepsilon$-free if $\varepsilon$ is not a vertex of $G$ : $\varepsilon \notin V_{G}$.

For $L \subseteq N^{*}$, we write $u \leq L$ if $u$ is prefix of a word of $L: \exists v(u v \in L)$. Given $\varepsilon$-free automata $G$ and $H$ with an injection $\phi: N_{G} \times N_{H} \longrightarrow N$ and a non-terminal $\# \in N-N_{G}$, we define the length superposition $G / \phi_{, \#} H$ of $G$ on $H$ as the following word automaton:

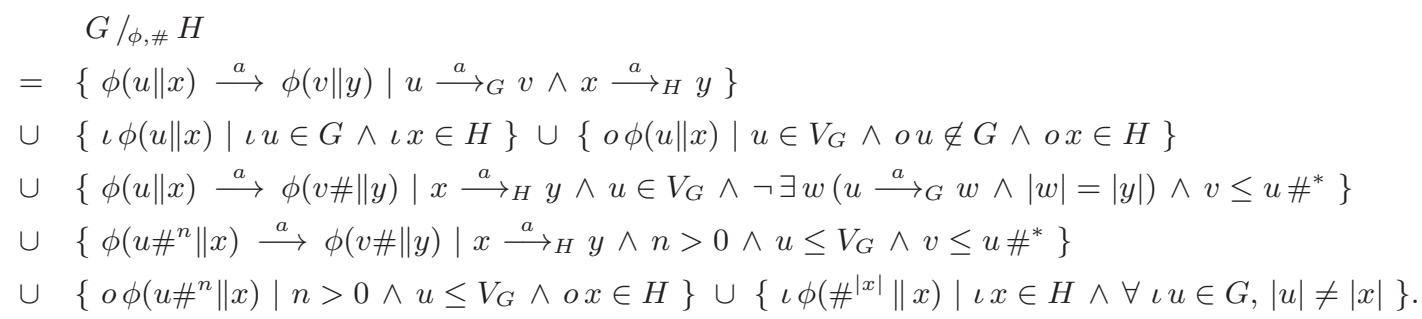

Since the coding $\phi$ is not essential, it will usually be omitted. Moreover, we will assume that \# is always a new non-terminal.

The definition of $G / H$ is done in order to follow in parallel and by length the paths of $G$ and $H$. When a transition of $H$ can not be length synchronized by $G$, a transition of $G / H$ leads to a copy of $H$ by marking the vertices by \#. Note that

$$
G, H \text { deterministic } \Longrightarrow G / H \text { deterministic. }
$$

As an example, we have $G \stackrel{f}{\longrightarrow} \ell \mathrm{d} H$ for the following $\varepsilon$-free deterministic automaton $G$ :

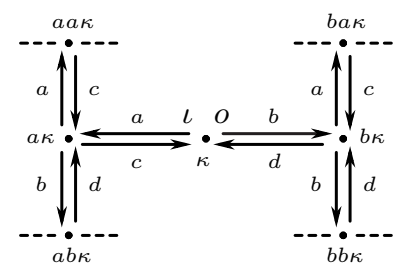

for the morphism $f(u \kappa)=\left.\right|^{|u|} \kappa$ for any $u \in\{a, b\}^{*}$ and for the following automaton $H$ :

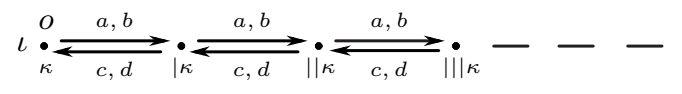

We represent below $(G / H)_{\iota, o}$ where any vertex $u$ stands for the word $u \|\left.\right|^{|u|-1} \kappa$. 


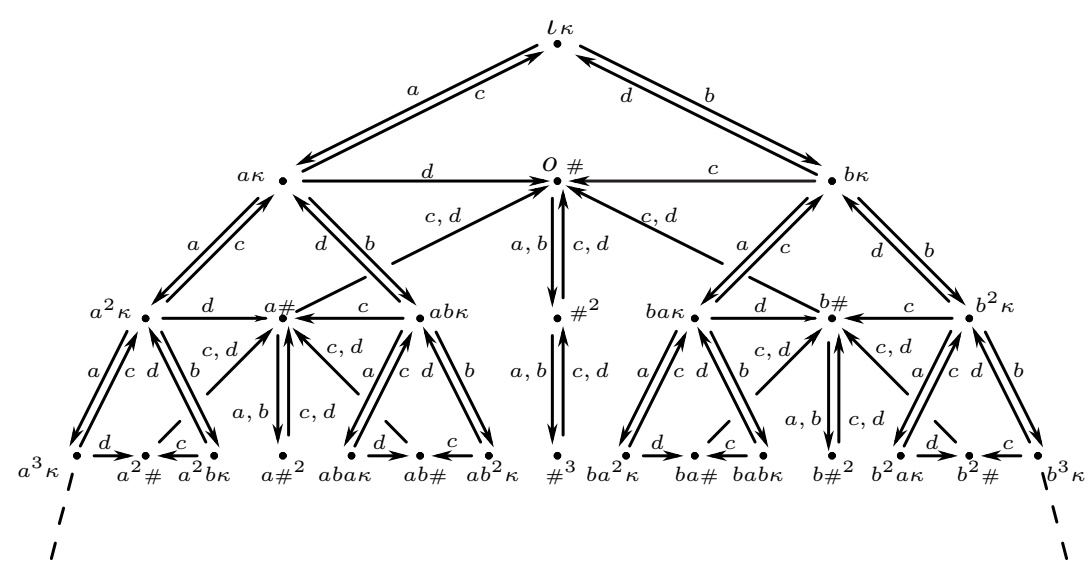

In order to avoid crossing edges, one can also represent this automaton by the following fractal picture:

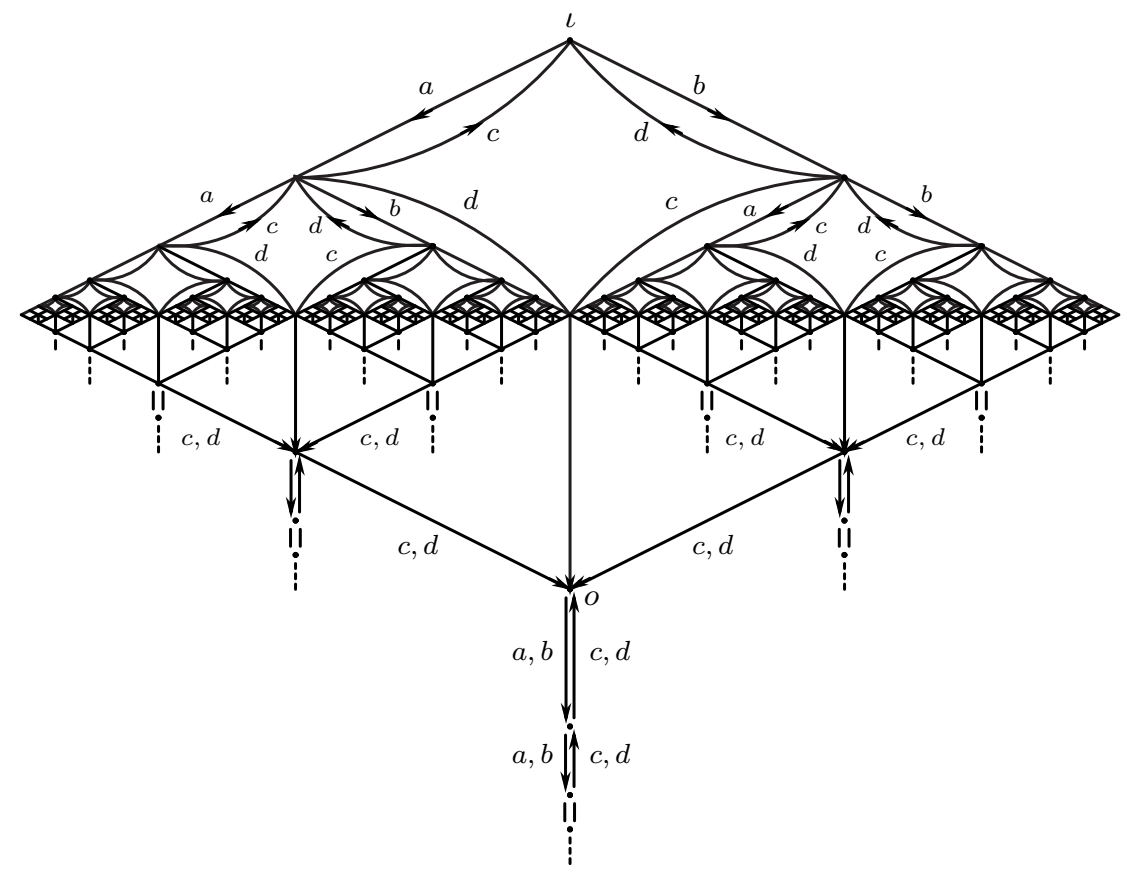

The length superposition gives the closure under difference.

- Lemma 12. For any $\varepsilon$-free automata $G, H,(G / H) \stackrel{\pi_{2}}{\longrightarrow} H$ and $\mathrm{L}(H)-\mathrm{L}(G) \subseteq \mathrm{L}(G / H)$. If $G \longrightarrow_{\ell} H$ and $(G / H) \stackrel{\pi_{2}}{\longrightarrow} H$ with $H$ unambiguous then $\mathrm{L}(G / H) \subseteq \mathrm{L}(H)-\mathrm{L}(G)$.

Proof.

i) Let us check that $(G / H) \stackrel{\pi_{2}}{\longrightarrow} H$. Let $s \stackrel{a}{\longrightarrow}_{G / H} t$.

So $s=u \| x$ and $t=v \| y$ with $x \stackrel{a}{\longrightarrow}_{H} y$. Thus $\pi_{2}(s)=x \stackrel{a}{\longrightarrow}_{H} y=\pi_{2}(t)$.

Let $c s \in G / H$. So $s=u \| x$ with $c x \in H$. In particular $c \pi_{2}(s)=c x \in H$.

ii) Let $a_{1} \ldots a_{n} \in \mathrm{L}(H)-\mathrm{L}(G)$ for some $n \geq 0$ and $a_{1}, \ldots, a_{n} \in T$.

Let us show that $a_{1} \ldots a_{n} \in \mathrm{L}(G / H)$.

There exists $x_{0}{\stackrel{a_{1}}{\longrightarrow}}_{H} x_{1} \ldots{\stackrel{a_{n}}{\longrightarrow}}_{H} x_{n}$ with $\iota x_{0}, o x_{n} \in H$.

Let $z_{i}=\left(\#^{\left|x_{i}\right|}, x_{i}\right)$ for any $0 \leq i \leq n$.

By definition of $G / H, z_{0}{\stackrel{a_{1}}{\longrightarrow}}_{G / H} z_{1} \ldots{\stackrel{a_{n}}{\longrightarrow}}_{G / H} z_{n}$ with $o z_{n} \in G / H$. 
We distinguish the two complementary cases below.

Case 1: $\neg \exists u_{0}\left(\iota u_{0} \in G \wedge\left|u_{0}\right|=\left|x_{0}\right|\right)$. So $\iota z_{0} \in G / H$ hence $a_{1} \ldots a_{n} \in \mathrm{L}(G / H)$.

Case 2: $\exists u_{0}\left(\iota u_{0} \in G \wedge\left|u_{0}\right|=\left|x_{0}\right|\right)$. Let $0 \leq m \leq n$ maximal such that

$$
u_{0} \stackrel{a_{1}}{\longrightarrow} G u_{1} \ldots \stackrel{a_{m}}{\longrightarrow} G u_{m} \text { with }\left|u_{1}\right|=\left|x_{1}\right|, \ldots,\left|u_{m}\right|=\left|x_{m}\right| \text {. }
$$

By definition of $G / H, u_{0}\left\|x_{0}{\stackrel{a_{1}}{\longrightarrow}}_{G / H} u_{1}\right\| x_{1} \ldots{\stackrel{a_{m}}{\longrightarrow}}_{G / H} u_{m} \| x_{m}$ and $\iota\left(u_{0} \| x_{0}\right) \in G / H$.

Case 2.1: $m=n$. As $a_{1} \ldots a_{n} \notin \mathrm{L}(G), o u_{n} \notin G$.

Thus $o\left(u_{n} \| x_{n}\right) \in G / H$ hence $a_{1} \ldots a_{n} \in \mathrm{L}(G / H)$.

Case 2.2: $m<n$.

Thus $u_{m}\left\|x_{m} \stackrel{a_{m+1}}{\longrightarrow} G / H u_{m+1}^{\prime} \#\right\| x_{m+1} \ldots{\stackrel{a_{m}}{\longrightarrow}}_{G / H} u_{n}^{\prime} \# \| x_{n}$ for some $u_{m+1}^{\prime}, \ldots, u_{n}^{\prime}$.

As $o x_{n} \in H$, we have $o\left(u_{n}^{\prime} \# \| x_{n}\right) \in G / H$ hence $a_{1} \ldots a_{n} \in \mathrm{L}(G / H)$.

iii) Assume that $G \stackrel{f}{\longrightarrow} \ell H$ and $(G / H) \stackrel{\pi_{2}}{\longrightarrow} \mathrm{d} H$ with $H$ unambiguous.

Let $w \in \mathrm{L}(G / H)$. Let us check that $w \in \mathrm{L}(H)-\mathrm{L}(G)$.

By Lemma $1, w \in \mathrm{L}(H)$. Assume that $w \in \mathrm{L}(G)$.

There is a path $u \stackrel{w}{\longrightarrow}_{G} v$ with $\iota u, o v \in G$. Thus $f(u) \stackrel{w}{\longrightarrow}_{H} f(v)$ with $\iota f(u), o f(v) \in H$. As $f$ is length-preserving, $u\left\|f(u) \stackrel{w}{\longrightarrow}_{G \| H} v\right\| f(v)$ with $\iota(u \| f(u)), o(v \| f(v)) \in G \| H$. Thus $u\left\|f(u) \stackrel{w}{\longrightarrow}_{G / H} v\right\| f(v)$ with $\iota(u \| f(u)) \in G / H$.

Furthermore $o \pi_{2}(v \| f(v))=o f(v) \in H$.

By Lemma 4, o $(v \| f(v)) \in G / H$. Thus ov $\notin G$ which is a contradiction.

Let us apply Lemma 12 restricted to deterministic automata with Proposition 9.

- Proposition 13. The language family $\ell \operatorname{dRec}_{\mathcal{F}}(H)=\ell \operatorname{Rec}_{\mathcal{F}_{\text {det }}}(H)$ is closed under difference when $H$ is deterministic and $\varepsilon$-free, and $\mathcal{F}$ is closed under $\|$ and $/$.

In general, the condition $(G / H) \stackrel{\pi_{2}}{\longrightarrow} \mathrm{d} H$ is necessary in Lemma 12. For instance, let us consider the following $\varepsilon$-free unambigous automaton $H$ :

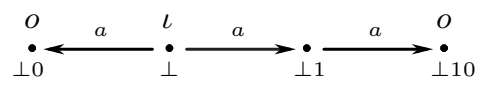

Here is the length superposition $(H / H)_{\iota}$ where any vertex $u, v$ represents $\perp u \| \perp v$.

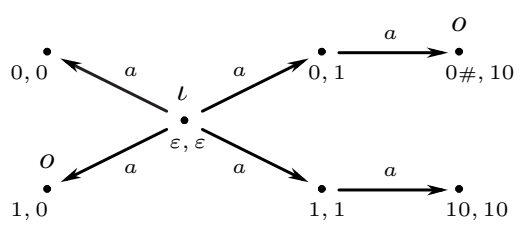

Thus $H / H$ is not deterministically reducible into $H$ and $\mathrm{L}(H / H)=\mathrm{L}(H)=\{a, a a\}$. In order to accept $\mathrm{L}(H)-\mathrm{L}(G)$ by length superposition when $G \longrightarrow \ell \mathrm{d} H$, we have to restrict to vertices of the trimmed automaton $G \| H$ and to vertices of the copies of $H$. We define the restricted length superposition $G / / H$ by

$$
G / / H=(G / H)_{\mid P} \text { for } P=V_{(G \| H)_{\iota, o}} \cup\left\{u \#^{|x|-|u|} \| x|| x|>| u \mid \wedge u \leq V_{G} \wedge x \in V_{H}\right\} .
$$

For the previous automaton $H$, the automaton $(H / / H)_{\iota}$ is the following:

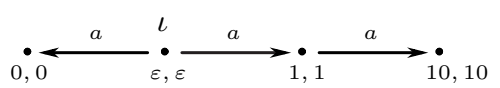

We get that $\mathrm{L}(H / / H)=\emptyset=\mathrm{L}(H)-\mathrm{L}(H)$. Such an example can be generalized.

Lemma 14. For any $\varepsilon$-free automata $G, H$ such that $G \longrightarrow \ell \mathrm{d} H$, we have

a) $H$ length-unambiguous $\Longrightarrow(G / / H) \longrightarrow \ell \mathrm{d} H$

b) $H$ unambiguous $\Longrightarrow \mathrm{L}(G / / H) \subseteq \mathrm{L}(H)-\mathrm{L}(G)$ 
c) $G$ trimmed and $H$ length-deterministic $\Longrightarrow \mathrm{L}(H)-\mathrm{L}(G) \subseteq \mathrm{L}(G / / H)$.

\section{Proof.}

Let $G \stackrel{f}{\longrightarrow} \ell \mathrm{d} H$ with $H$ length-unambiguous.

i) Let $u \| x$ be a vertex of $G / / H$ with $u$ a vertex of $G$.

By definition of $G / / H, u \| x$ is a vertex of $(G \| H)_{\iota, o}$.

Note that $H \stackrel{i d}{\longrightarrow} \ell \mathrm{d} H$ for the identity mapping $i d$ on $V_{H}$.

By Lemma 8 (b), we have $f(u)=i d(x)=x$.

ii) Let us prove that $(G / / H) \stackrel{\pi_{2}}{\longrightarrow} \ell \mathrm{d} H$. By Lemma 12 , we have $(G / / H) \stackrel{\pi_{2}}{\longrightarrow} \ell H$.

It remains to show that the morphism $\pi_{2}$ is deterministic.

Let $\iota s, \iota t \in G / / H$ with $\pi_{2}(s)=\pi_{2}(t)$. We have to check that $s=t$.

Note that $s, t$ can not be of the form $u \#^{n} \| x$ for $\varepsilon \neq u \leq V_{G}$ and $n>0$.

It remains the complementary cases below.

Case 1: $s, t$ are vertices of $(G \| H)_{\iota, o}$.

By (i), we have $s=u \| f(u)$ and $t=v \| f(v)$ for some $\iota u, \iota v \in G$.

Furthermore $f(u)=\pi_{2}(s)=\pi_{2}(t)=f(v)$.

As $f$ is deterministic, we get $u=v$ hence $s=t$.

Case 2: $s$ is a vertex of $(G \| H)_{\iota, o}$ and $t=\left(\#^{|x|}, x\right)$ for some vertex $x$ of $H$.

By (i), we have $s=u \| f(u)$ for some $\iota u \in G$.

Furthermore $f(u)=\pi_{2}(s)=\pi_{2}(t)=x$. In particular $|u|=|f(u)|=|x|$.

By definition of $G / / H, \iota t \notin H$ which is a contradiction, hence Case 2 is impossible.

Case 3: $t$ is a vertex of $(G \| H)_{\iota, o}$ and $s=\left(\#^{|x|}, x\right)$ for some vertex $x$ of $H$.

By symmetry of $s, t$ and by Case 2 , this case is also impossible

Case 4: $s=\left(\#^{|x|}, x\right)$ and $t=\left(\#^{|y|}, y\right)$ for some vertices $x, y$ of $H$.

Thus $x=\pi_{2}(s)=\pi_{2}(t)=y$ hence $s=t$.

Let $r \stackrel{a}{\longrightarrow}_{G / / H} s$ and $r \stackrel{a}{\longrightarrow}_{G / / H} t$ with $\pi_{2}(s)=\pi_{2}(t)$. We have to check that $s=t$.

We have the complementary cases below knowing that the remaining cases are not possible.

Case 1: $s, t$ are vertices of $(G \| H)_{\iota, o}$.

By (i), we have $r=u\|f(u), s=v\| f(v), t=w \| f(w)$ for $u \stackrel{a}{\longrightarrow} G$ and $u \stackrel{a}{\longrightarrow}_{G} w$.

Furthermore $f(v)=\pi_{2}(s)=\pi_{2}(t)=f(w)$.

As $f$ is deterministic, we get $v=w$ hence $s=t$.

Case 2: $s=v \# \| y$ and $t=w \# \| z$.

So $v \leq u \#^{*}$ and $w \leq u \#^{*}$ for some $u \leq V_{G}$.

Furthermore $y=\pi_{2}(s)=\pi_{2}(t)=z$. So $|v \#|=|y|=|z|=|w \#|$.

Thus $v=w$ hence $s=t$.

iii) Suppose that $H$ is unambiguous. Let us prove that $\mathrm{L}(G / / H) \subseteq \mathrm{L}(H)-\mathrm{L}(G)$.

Let $w \in \mathrm{L}(G / / H)$. By Lemma 12 (a) and $1, w \in \mathrm{L}(H)$.

Assume that $w \in \mathrm{L}(G)$. There exists $u \stackrel{w}{\longrightarrow} G$ with $\iota u, o v \in G$.

So $u\left\|f(u) \stackrel{w}{\longrightarrow}_{G / / H} v\right\| f(v)$ with $\iota(u \| f(u)) \in G / / H$.

Furthermore $o \pi_{2}(v \| f(v))=o f(v) \in H$.

By (ii) and Lemma 4, o(v\|f(v)) $\in G / / H$ which is a contradiction.

iv) Suppose that $G$ is trimmed and $H$ is length-deterministic.

Let us prove that $\mathrm{L}(H)-\mathrm{L}(G) \subseteq \mathrm{L}(G / / H)$.

Let $a_{1} \ldots a_{n} \in \mathrm{L}(H)-\mathrm{L}(G)$ for some $n \geq 0$ and $a_{1}, \ldots, a_{n} \in T$.

Let us show that $a_{1} \ldots a_{n} \in \mathrm{L}(G / / H)$.

There exists $x_{0}{\stackrel{a_{1}}{\longrightarrow}}_{H} x_{1} \ldots{\stackrel{a_{n}}{\longrightarrow}}_{H} x_{n}$ with $\iota x_{0}, o x_{n} \in H$.

Let $z_{i}=\left(\#^{\left|x_{i}\right|}, x_{i}\right)$ for any $0 \leq i \leq n$. 
By definition of $G / / H, z_{0}{\stackrel{a_{1}}{\longrightarrow}}_{G / / H} z_{1} \ldots{\stackrel{a_{n}}{\longrightarrow}}_{G / / H} z_{n}$ with $o z_{n} \in G / / H$.

We distinguish the two complementary cases below.

Case 1: $\neg \exists u_{0}\left(\iota u_{0} \in G \wedge\left|u_{0}\right|=\left|x_{0}\right|\right)$. So $\iota z_{0} \in G / / H$ hence $a_{1} \ldots a_{n} \in \mathrm{L}(G / / H)$.

Case 2: $\exists u_{0}\left(\iota u_{0} \in G \wedge\left|u_{0}\right|=\left|x_{0}\right|\right)$. Let $0 \leq m \leq n$ maximal such that

$$
u_{0}{\stackrel{a_{1}}{\longrightarrow}}_{G} u_{1} \ldots{\stackrel{a_{m}}{\longrightarrow}}_{G} u_{m} \text { with }\left|u_{1}\right|=\left|x_{1}\right|, \ldots,\left|u_{m}\right|=\left|x_{m}\right| .
$$

Thus $u_{0}\left\|x_{0}{\stackrel{a_{1}}{\longrightarrow}}_{G \| H} u_{1}\right\| x_{1} \ldots{\stackrel{a_{m}}{\longrightarrow}}_{G \| H} u_{m} \| x_{m}$ and $\iota\left(u_{0} \| x_{0}\right) \in G \| H$.

As $H$ is length-deterministic and by Lemma 8 (a), $f\left(u_{i}\right)=i d\left(x_{i}\right)=x_{i}$ for any $0 \leq i \leq m$.

As $G$ is trimmed, there exists a path $u_{m} \longrightarrow_{G}^{*} u^{\prime}$ with $o u^{\prime} \in G$.

Thus $\left(u_{m} \| x_{m}\right)=\left(u_{m} \| f\left(u_{m}\right)\right) \longrightarrow_{G \| H}^{*}\left(u^{\prime} \| f\left(u^{\prime}\right)\right)$ with $o\left(u^{\prime} \| f\left(u^{\prime}\right)\right) \in G \| H$.

It follows that $u_{m} \| x_{m}$ is a vertex of $(G \| H)_{\iota, o}$ hence a vertex of $G / / H$.

Case 2.1: $m=n$. As $a_{1} \ldots a_{n} \notin \mathrm{L}(G), o u_{n} \notin G$.

Thus $o\left(u_{n} \| x_{n}\right) \in G / / H$ hence $a_{1} \ldots a_{n} \in \mathrm{L}(G / / H)$.

Case 2.2: $m<n$.

Thus $u_{m}\left\|x_{m} \stackrel{a_{m+1}}{\longrightarrow} G / / H u_{m+1}^{\prime} \#\right\| x_{m+1} \ldots{\stackrel{a_{m}}{\longrightarrow}}_{G / / H} u_{n}^{\prime} \# \| x_{n}$ for some $u_{m+1}^{\prime}, \ldots, u_{n}^{\prime}$.

As $o x_{n} \in H$, we have $o\left(u_{n}^{\prime} \# \| x_{n}\right) \in G / / H$ hence $a_{1} \ldots a_{n} \in \mathrm{L}(G / / H)$.

Let us apply Lemma 14 with Proposition 11.

- Proposition 15. The language family $\ell \operatorname{dRec}_{\mathcal{F}}(H)$ is closed under difference when $H$ is unambiguous, $\varepsilon$-free and length-deterministic, and $\mathcal{F}$ is closed under เo-restriction, $\|$ and //.

Propositions 11 and 15 give Boolean algebras by length-preserving deterministic recognizability.

- Theorem 16. The language family $\ell \operatorname{dRec}_{\mathcal{F}}(H)$ is a Boolean algebra relative to $\mathrm{L}(H)$ for any automata family $\mathcal{F}$ closed under the operations $\|$ and $/ \mid$ and $\iota 0-r e s t r i c t i o n$, and for any automaton $H$ in $\mathcal{F}$ which is unambiguous, $\varepsilon$-free and length-deterministic.

The closure under $\iota$-restriction is not satisfied for general automata families because the closure under accessibility and co-accessibility is required. This can then be avoided by restricting to deterministic automata through Propositions 9 and 13.

- Theorem 17. The language family $\ell \operatorname{dRec}_{\mathcal{F}}(H)=\ell \operatorname{Rec}_{\mathcal{F}_{\text {det }}}(H)$ is a Boolean algebra relative to $\mathrm{L}(H)$ for any automata family $\mathcal{F}$ closed under the operations $\|$ and /, and for any automaton $H$ in $\mathcal{F}$ which is deterministic and $\varepsilon$-free.

We apply these two theorems for general automata families.

\section{Boolean algebras of context-free languages}

A general way of accepting context-free languages is through suffix automata. We prove that this automaton family is closed under previous operations to get Boolean algebras of context-free languages by Theorem 16.

An elementary suffix automaton is an automaton of the form:

$$
W(u \stackrel{a}{\longrightarrow} v)=\{w u \stackrel{a}{\longrightarrow} w v \mid w \in W\} \text { where } W \in \operatorname{Reg}\left(N^{*}\right), u, v \in N^{*}, a \in T \cup\{\iota, o\} .
$$

A suffix automaton is a finite union of elementary suffix automata. The family $\mathcal{S}$ tack of suffix automata defines the family $\mathcal{L}(\mathcal{S}$ tack $)$ of context-free languages.

For instance, the previous 'fractal' automaton $F r$ is in $\mathcal{S}$ tack: Denoting $(\kappa, \kappa)$ by $\kappa,(\#, \kappa)$ by $\#_{\kappa}$, and $(x, \mid)$ by $x$ for any $x \in\{a, b, \#\}, F r$ is the union of the following elementary suffix automata: 


$$
\begin{array}{llll}
\{a, b\}^{*}(\kappa \stackrel{a}{\longrightarrow} a \kappa) & \{a, b\}^{*}(\kappa \stackrel{b}{\longrightarrow} b \kappa) & \{a, b\}^{*}(a \kappa \stackrel{c}{\longrightarrow} \kappa) & \{a, b\}^{*}(b \kappa \stackrel{d}{\longrightarrow} \kappa) \\
\{a, b\}^{*}\left(a \kappa \stackrel{d}{\longrightarrow} \#_{\kappa}\right) & \{a, b\}^{*}\left(b \kappa \stackrel{c}{\longrightarrow} \#_{\kappa}\right) & \{a, b\}^{*}\left(a \#_{\kappa} \stackrel{c, d}{\longrightarrow} \#_{\kappa}\right) & \{a, b\}^{*}\left(b \#_{\kappa} \stackrel{c, d}{\longrightarrow} \#_{\kappa}\right) \\
\{a, b\}^{*} \#^{*}\left(\#_{\kappa} \stackrel{a, b}{\longrightarrow} \# \#_{\kappa}\right) & \{a, b\}^{*} \#^{*}\left(\# \#_{\kappa} \stackrel{c, d}{\longrightarrow} \#_{\kappa}\right) & \{\varepsilon\}(\kappa \stackrel{\iota}{\longrightarrow} \kappa) & \{\varepsilon\}(\# \stackrel{o}{\longrightarrow} \#)
\end{array}
$$

This general form of suffix automata allows to get their closure under the previous operations.

- Lemma 18. The family Stack is closed under ıo-restriction, ||, / and //.

\section{Proof.}

i) $\mathcal{S}$ tack is closed under regular restriction which is distributive over union and satisfies $W(u \stackrel{a}{\longrightarrow} v)_{\mid P}=\{w u \stackrel{a}{\longrightarrow} w v \mid w \in W \wedge w u, w v \in P\}=\left(W \cap P u^{-1} \cap P v^{-1}\right)(u \stackrel{a}{\longrightarrow} v)$ where $P u^{-1}=\{v \mid v u \in P\}$ is the right residual of $P \subseteq N^{*}$ by $u \in N^{*}$.

Given an automaton $G$ in $\mathcal{S}$ tack and a letter $\star$ in $T$, the graph $\left\{u \stackrel{\star}{\longrightarrow} v \mid u \longrightarrow_{G}^{*} v\right\}$ is in $\mathcal{S}$ tack (Proposition 3.18 in [2]). In particular $\longrightarrow_{G}^{*}$ is a rational relation: it is recognized by a finite transducer. Thus, the set of vertices deriving from or to a regular vertex subset remains regular. Hence $\mathcal{S}$ tack is closed under $\iota$-restriction.

ii) $\mathcal{S}$ tack is closed under $\|$ since this operation is distributive over union, and we have

$$
\begin{aligned}
& W(u \stackrel{a}{\longrightarrow} v) \| Z(x \stackrel{a}{\longrightarrow} y) \\
= & \{(w u \| z x) \stackrel{a}{\longrightarrow}(w v \| z y) \mid w \in W \wedge z \in Z \wedge(|w u|=|z x| \wedge|w v|=|z y|)\} \\
= & \{(w u \| z x) \stackrel{a}{\longrightarrow}(w v \| z y) \mid w \in W \wedge z \in Z \wedge(|u|-|x|=|z|-|w|=|v|-|y|)\} .
\end{aligned}
$$

So $W(u \stackrel{a}{\longrightarrow} v) \| Z(x \stackrel{b}{\longrightarrow} y)=\emptyset$ if $a \neq b$ or $|u|-|v| \neq|x|-|y|$, and otherwise is equal to

$$
\begin{aligned}
& \bigcup_{s \in N^{|x|-|u|}}\left(W s^{-1} \| Z\right) \cdot((s u \| x) \stackrel{a}{\longrightarrow}(s v \| y)) \text { for }|u| \leq|x| \\
& \bigcup_{s \in N^{|u|-|x|}}\left(W \| Z s^{-1}\right) \cdot((u \| s x) \stackrel{a}{\longrightarrow}(v \| s y)) \text { for }|u|>|x| .
\end{aligned}
$$

Furthermore for $G, G^{\prime} \in \mathcal{S}$ tack, $I_{G \| G^{\prime}}=I_{G} \| I_{G^{\prime}}$ remains regular and is described by the rule $\left(I_{G} \| I_{G^{\prime}}\right) \cdot(\varepsilon \stackrel{\iota}{\longrightarrow} \varepsilon)$. It is the same for $O_{G \| G^{\prime}}=O_{G} \| O_{G^{\prime}}$.

iii) Let us show that $\mathcal{S}$ tack is closed under /. As $G /\left(H \cup H^{\prime}\right)=G / H \cup G / H^{\prime}$, it remains to consider $G / Z(x \stackrel{a}{\longrightarrow} y)$ for $G=\bigcup_{i=1}^{n} W_{i}\left(u_{i} \stackrel{a_{i}}{\longrightarrow} v_{i}\right)$. Let us define the language

$$
L=\bigcup\left\{W_{i} \cdot u_{i}\left|1 \leq i \leq n \wedge a_{i}=a \wedge\right| u_{i}|-| v_{i}|=| x|-| y \mid\right\} .
$$

Let us check that

$$
\left(V_{G}-L\right) \| Z x=\left\{s \| z x\left|s \in V_{G} \wedge z \in Z \wedge\right| s|=| z x \mid \wedge \neg \exists t(s \stackrel{a}{\longrightarrow} t \wedge|t|=|z y|)\right\} .
$$

Let $s \in V_{G}$ and $z \in Z$ such that $|s|=|z x|$. We have to show that

$$
s \in L \Longleftrightarrow \exists t\left(s \stackrel{a}{\longrightarrow}_{G} t \wedge|t|=|z y|\right) .
$$

$\Longrightarrow$ : Assume that $s \in L$.

There exists $1 \leq i \leq n$ and $w \in W_{i}$ such that $s=w u_{i}$ and $a_{i}=a$ and $\left|u_{i}\right|-\left|v_{i}\right|=|x|-|y|$. Hence $s \stackrel{a}{\longrightarrow} w v_{i}$ with $\left|w v_{i}\right|=|w|+\left|u_{i}\right|+|y|-|x|=|s|+|y|-|x|=|z y|$.

$\Longleftarrow$ : Suppose there exists $t$ such that $s \stackrel{a}{\longrightarrow}_{G} t$ and $|t|=|z y|$.

Hence there exists $1 \leq i \leq n$ and $w \in W_{i}$ such that $a_{i}=a, s=w u_{i}$ and $t=w v_{i}$.

As $|z x|=|s|$ and $|z y|=\left|w v_{i}\right|$, we get $\left|w v_{i}\right|-|y|=|z|=|s|-|x|$.

Thus $\left|w v_{i} x\right|=|s y|=\left|w u_{i} y\right|$ i.e. $\left|u_{i} y\right|=\left|v_{i} x\right|$. So $s=w u_{i} \in L$.

Thus the following subgraph of $G / H$ :

$$
\left\{u\|x \stackrel{a}{\longrightarrow} v \#\| y \mid x \stackrel{a}{\longrightarrow}_{H} y \wedge u \in V_{G} \wedge \neg \exists w(u \stackrel{a}{\longrightarrow} G \wedge \wedge|w|=|y|) \wedge v \leq u \#^{*}\right\}
$$

corresponding to the 'stall' of $G$ w.r.t. $H$, is equal to the following suffix automaton:

$$
\left(V_{G}-L\right) \cdot\left(\varepsilon \stackrel{a}{\longrightarrow} \#^{|y|-|x|}\right) \| Z .(x \stackrel{a}{\longrightarrow} y) \text { for }|x|<|y|
$$

otherwise $|x| \geq|y|$ and by union on $1 \leq i \leq n$ with $W=W_{i}$ and $u \in\left\{u_{i}, v_{i}\right\}$, 
if $|u|>|x|-|y|$ we take the suffix automaton:

$$
\left(W-L u^{-1}\right) \cdot(u \stackrel{a}{\longrightarrow} v \#) \| Z .(x \stackrel{a}{\longrightarrow} y) \text { for } v<u \text { and }|u|-|v \#|=|x|-|y|
$$

and if $|u| \leq|x|-|y|$, having $|u|=|x|$ we get $y=\varepsilon$ and we take the suffix automaton:

$\left(W-L u^{-1}\right) s^{-1}$. (su $\left.\stackrel{a}{\longrightarrow} \#\right) \| Z$. $(x \stackrel{a}{\longrightarrow} \varepsilon)$ for any suffix letter $s$ of $W$.

Similarly denoting by $P_{G}$ the set of prefixes of $V_{G}$, the following subgraph of $G / H$ :

$$
\left\{u \#^{n}\|x \stackrel{a}{\longrightarrow} v \#\| y \mid x \stackrel{a}{\longrightarrow}_{H} y \wedge n>0 \wedge u \leq V_{G} \wedge v \leq u \#^{*}\right\}
$$

is equal to the following suffix automaton:

$$
P_{G} \#^{+} .(\varepsilon \stackrel{a}{\longrightarrow} \#|y|-|x|) \| Z .(x \stackrel{a}{\longrightarrow} y) \text { for }|x| \leq|y|
$$

otherwise $|x|>|y|$ and the automaton is equal to the unions of the following automata:

$$
\left(P_{G} \#^{+}\right) u^{-1} \cdot(u \stackrel{a}{\longrightarrow} \#) \| Z .(x \stackrel{a}{\longrightarrow} y) \text { for } u \in N_{G}^{*} \#^{+} \text {and }|u|=|x|-|y|+1 .
$$

Finally, the other subgraphs of $G / H$ are described as before.

With (i), it follows that $\mathcal{S}$ tack is also closed under //.

Let us apply Theorem 16 with Lemma 18.

- Proposition 19. The family $\ell \operatorname{dRec}_{\mathcal{S} \text { tack }}(H)$ is a Boolean algebra relative to $\mathrm{L}(H)$ for any unambiguous, $\varepsilon$-free and length-deterministic automaton $H$.

In particular, we obtain again that $\ell \operatorname{dRec}_{\mathcal{S} \text { tack det }}(H)$ for $H$ deterministic, is a relative Boolean algebra [7].

A well-known relative Boolean algebra is the family $\ell \operatorname{dRec}_{\mathcal{S} \text { tack }}\left(\operatorname{Inp}\left(T_{-1}, T_{0}, T_{1}\right)\right)$ of inputdriven languages according to the triple $\left(T_{-1}, T_{0}, T_{1}\right)$ of finite disjoint subsets of $T$ [6]. Adding the loops labeled in $T_{-1}$ on the initial vertex $\kappa$ of $\operatorname{Inp}\left(T_{-1}, T_{0}, T_{1}\right)$, we get the visibly automaton $\operatorname{Vis}\left(T_{-1}, T_{0}, T_{1}\right)=\operatorname{Inp}\left(T_{-1}, T_{0}, T_{1}\right) \cup\left\{\kappa \stackrel{a}{\longrightarrow} \kappa \mid a \in T_{-1}\right\}$ accepting $\mathrm{L}\left(\operatorname{Vis}\left(T_{-1}, T_{0}, T_{1}\right)\right)=\left(T_{-1} \cup T_{0} \cup T_{1}\right)^{*}$, and $\ell \operatorname{dRec}_{\mathcal{S}_{\text {tack }}}\left(\operatorname{Vis}\left(T_{-1}, T_{0}, T_{1}\right)\right)$ is the Boolean algebra of visibly pushdown languages according to $\left(T_{-1}, T_{0}, T_{1}\right)$ [1].

Note that we can enhance the visibility of pushdown automata by taking a mapping \|\| from a finite subset $T_{\|\|} \subset T$ to $\mathbb{Z}$, by taking $\mid, \kappa \in N$, and by defining the automaton

$$
\mathrm{Vis}_{\|\|}=\left\{\left.\left.\right|^{n} \kappa \stackrel{a}{\longrightarrow}\right|^{\max (0, n+\|\mathrm{a}\|)} \mid n \geq 0 \wedge a \in T_{\|\|}\right\} \cup\{\iota \kappa\} \cup\left\{\left.o\right|^{n} \kappa \mid n \geq 0\right\}
$$

In particular $\operatorname{Vis}\left(T_{-1}, T_{0}, T_{1}\right)=\operatorname{Vis}_{\|\|}$for $T_{\|\|}=T_{-1} \cup T_{0} \cup T_{1}$ with $\|a\|=i$ for any $a \in T_{i}$ and $i \in\{-1,0,1\}$. For any \|\|$, \mathrm{L}\left(\operatorname{Vis}_{\|\|}\right)=T_{\|\|}^{*}$ and $\ell \operatorname{dRec}_{\mathcal{S} \operatorname{tack}}\left(\operatorname{Vis}_{\|\|}\right)=$ $\ell \operatorname{Rec}_{\mathcal{S t a c k}_{\mathrm{det}}}\left(\operatorname{Vis}_{\|\|}\right)$is a Boolean algebra.

We further increase the pushdown visibility by taking $\mid, \dagger, \kappa \in N$ and the recognizer

$$
2 \mathrm{Vis}_{\|\|}=\left\{\left.\left.\right|^{n} \kappa \stackrel{a}{\longrightarrow}\right|^{n+\|\mathrm{a}\|} \mid n \in \mathbb{Z} \wedge a \in T_{\|\|}\right\} \cup\{\iota \kappa\} \cup\left\{\left.o\right|^{n} \kappa \mid n \in \mathbb{Z}\right\}
$$

where $\left.\right|^{-n}=\dagger^{n}$ for any $n>0$. Thus $\operatorname{ldRec}_{\mathcal{S} \text { tack }}\left(\operatorname{Vis}_{\|\|}\right)$is still a Boolean algebra.

Note that Proposition 19 also applies to non-deterministic recognizers like the previous unambiguous automaton $U n$ which is also $\varepsilon$-free and length-deterministic.

Proposition 19 may also be restricted to the family of counter automata.

\section{Boolean algebras of context-sensitive languages}

A simple way to define context-sensitive languages is through the synchronized relations of bounded length difference.

An elementary bounded synchronized automaton is an automaton of the form:

$R(u \stackrel{a}{\longrightarrow} v)=\{x u \stackrel{a}{\longrightarrow} y v \mid(x, y) \in R\}$ for $R \in \operatorname{Reg}\left((N \times N)^{*}\right), u, v \in N^{*}, a \in T \cup\{\iota, o\}$.

A bounded synchronized automaton is a finite union of elementary bounded synchonized automata. The family $\mathcal{S}$ ync of bounded synchronized automata accepts the family $\mathcal{L}(\mathcal{S}$ ync $)$ 
of context-sensitive languages [8].

Similarly to the proof of Lemma 18, we get that $\mathcal{S} y n c$ is closed under $\|$ and /. However, $\mathcal{S}$ ync is not closed under $\iota$-restriction, nor closed under // because the set of vertices accessible from a given vertex for a bounded synchronized automaton is not necessarily regular (and also not effective). Nevertheless and by restricting to deterministic recognizers, we can apply Theorem 17.

- Proposition 20. The family $\ell \operatorname{dRec}_{\mathcal{S} \text { ync }}(H)=\ell \operatorname{Rec}_{\mathcal{S}_{\mathrm{ync}_{\mathrm{det}}}}(H)$ is a Boolean algebra relative to $\mathrm{L}(H)$ for any deterministic and $\varepsilon$-free automaton $H$.

Thus $\ell \mathrm{dRec}_{\mathcal{S}_{\mathrm{ync}}}\left(\operatorname{Inp}\left(T_{-1}, T_{0}, T_{1}\right)\right)$ defines the Boolean algebra relative to $\mathrm{L}\left(\operatorname{Inp}\left(T_{-1}, T_{0}, T_{1}\right)\right)$ of bounded synchronized input-driven languages w.r.t. to $\left(T_{-1}, T_{0}, T_{1}\right)$. Likewise we have the Boolean algebra $\ell \operatorname{dRec}_{\mathcal{S}_{\mathrm{ync}}}\left(\mathrm{Vis}_{\|} \|\right)$of bounded synchronized visibly languages w.r.t. \| \|. Theorem 17 can be applied to many other automata families, as for example the family of vector addition systems (or Petri nets) with regular contexts.

In conclusion, the deterministic length recognizability allows to obtain Boolean algebras using automata families and recognizers. We have applied it to suffix automata and bounded synchronized automata but one can use it on any automata family closed under length synchronization, length superposition and trimmed restriction.

\section{References}

1 R. Alur and P. Madhusudan, Visibly pushdown languages, $36^{\text {th }}$ STOC, ACM Proceedings, L. Babai (Ed.), 202-211 (2004).

2 D. Caucal, On infinite transition graphs having a decidable monadic theory, $23^{\text {rd }}$ ICALP, LNCS 1099, F. Meyer auf der Heide, B. Monien (Eds.), 194-205 (1996).

3 D. Caucal Boolean algebras of unambiguous context-free languages, $28^{\text {th }}$ FSTTCS, Dagstuhl Research Server, R. Hariharan, M. Mukund, V. Vinay (Eds.) (2008).

4 D. Caucal and C. Rispal, Recognizability for automata, $22^{\text {nd }}$ DLT, to appear in LNCS, M. Hoshi, S. Seki (Eds.) (2018).

5 S. Eilenberg, Algèbre catégorique et théorie des automates, Institut H. Poincaré (1967), and Automata, languages and machines, Vol. A, Academic Press, New-York (1974).

6 K. Mehlhorn, Pebbling mountain ranges and its application to DCFL recognition, $7^{\text {th }}$ ICALP, LNCS 85, J. de Bakker, J. van Leeuwen (Eds.), 422-432 (1980).

7 D. Nowotka and J. Srba, Height-deterministic pushdown automata, $32^{\text {nd }}$ MFCS, LNCS 4708, L. Kucera, A. Kucera (Eds.), 125-134 (2007).

8 C. Rispal, The synchronized graphs trace the context-sensitive languages. Electr. Notes Theor. Comput. Sci. 68(6), 55-70 (2002).

9 W. Thomas, Uniform and nonuniform recognizability, Theoretical Computer Science 292, 299-316 (2003). 